\title{
Severe pneumonia due to infection with Candida krusei in a case of suspected Middle East respiratory syndrome: A case report and literature review
}

\author{
MINGMING TAN, JUNWEI WANG, PEIYANG HU, BIN WANG, WANGHUA XU and JIAO CHEN \\ Emergency Department, Tiantai County People's Hospital, Taizhou, Zhejiang 317200, P.R. China
}

Received April 30, 2015; Accepted May 25, 2016

DOI: $10.3892 /$ etm.2016.3892

\begin{abstract}
Candida krusei (C. krusei) pneumonia is a rare infection that is frequently associated with a poor outcome. The present study reports an unusual case of $C$. krusei pneumonia that was initially suspected to be a Middle East respiratory syndrome (MERS) case. A 64-year-old Saudi Arabian male patient was admitted to our hospital with complaints of cough and dyspnea that persisted for 6 days. The patient presented fever (oral temperature, $38.5^{\circ} \mathrm{C}$ ) and slight tachypnea (25 respirations/min). A chest computerized tomography demonstrated unclear lung fields, diffuse pathological changes in the two lungs and multiple lymphadenectasis in the retrocaval and para-aortic arch area. The patient received $95-98 \%$ oxygen $(6 \mathrm{l} / \mathrm{min})$ for $24 \mathrm{~h}$, as well as sulbactam sodium/cefoperazone sodium (1:1) injection (3.0 g) every $12 \mathrm{~h}$, oral oseltamivir capsules $(75 \mathrm{mg} /$ time $)$ twice a day, medaron injection (80 mg/time) and $750 \mathrm{ml}$ fluid infusion; however, he succumbed to the disease on day 2 after admission. The infection was diagnosed by sputum smear and culture subsequent to patient mortality. A sputum smear showed a large fungal infection and sputum culture revealed the presence of $C$. krusei infection. Serum procalcitonin concentrations were $4.73 \mu \mathrm{g} / \mathrm{l}$ and $7.23 \mu \mathrm{g} / 1$ on days 2 and 3 after admission, respectively. In conclusion, the diagnosis of Candida pneumonia should be strongly considered in the presence of growth of Candida from a sputum culture and based on a suggestive computed tomography image. Tumescent diaphragmatic lymph nodes may also be an important symptom of Candida pneumonia. Treatment should be initiated immediately to improve tissue oxygenation, restore cardiovascular function and improve other organ functions.
\end{abstract}

Correspondence to: Dr Junwei Wang, Emergency Department Tiantai County People's Hospital, 335 Laodong Road, Taizhou, Zhejiang 317200, P.R. China

E-mail: junweiwangjwwj@163.com

Key words: Candida krusei, Candida pneumonia, PCT

\section{Introduction}

Pneumonia due to Candida infection is rare and frequently associated with a fatal outcome, with a mortality rate up to $70 \%$ (1-3). It occurs predominantly in immunocompromised patients, or patients receiving broad-spectrum antibiotic therapy (4). Haron et al (5) revealed an incidence of $0.4 \%$ among 7,725 patients (1), which is similar to Masur's observations several years before (0.23\%). Candida pneumonia was first described by Nils Rosén von Rosenstein in 1984 (6), and later reported by Castellani in 1927 (7). However, only a limited number of cases have since been reported in the English literature (8). In search of the PubMed database (http://www.ncbi.nlm.nih.gov/pubmed accessed 14 May, 2009), 91 cases of Candida pneumonia confirmed by histopathology (1-3,8-10) were identified. In particular, Candida krusei (C. krusei) infection is less frequently encountered in clinical mycology compared with other species of Candida pathogens, with the first case of $C$. krusei infection reported in $2002(3,11)$.

The diagnosis of Candida pneumonia is one of the most challenging of all Candida infections to diagnose (12), not only because of its rare occurrence, but also its non-specific clinical manifestation, with fever and tachypnea being the most common symptoms and cough, expectoration of purulent secretions, hemoptysis, and chest pain only present occasionally (13). Making a convincing diagnosis of Candida pneumonia is only truly possible on the basis of a histopathological examination of samples $(14,15)$. MERS, a viral respiratory infection caused by the novel MERS-CoV pathogen, also had common symptoms with Candida pneumonia, including fever, cough and shortness of breath (16).

The present study reported a case of C. krusei pneumonia in a 64-year-old male patient, who was initially suspected with Middle East respiratory syndrome (MERS).

\section{Case report}

A 64-year-old Saudi Arabian male patient was admitted to Tiantai County People's Hospital (Taizhou, China) in December 2014 with complaints of cough and dyspnea that had persisted for 6 days. Written informed consent was obtained from the patient. The patient initially presented abrupt onset of paroxysmal cough without evident inducements, accompanied by chest tightness, tachypnea and slight fever; however, 

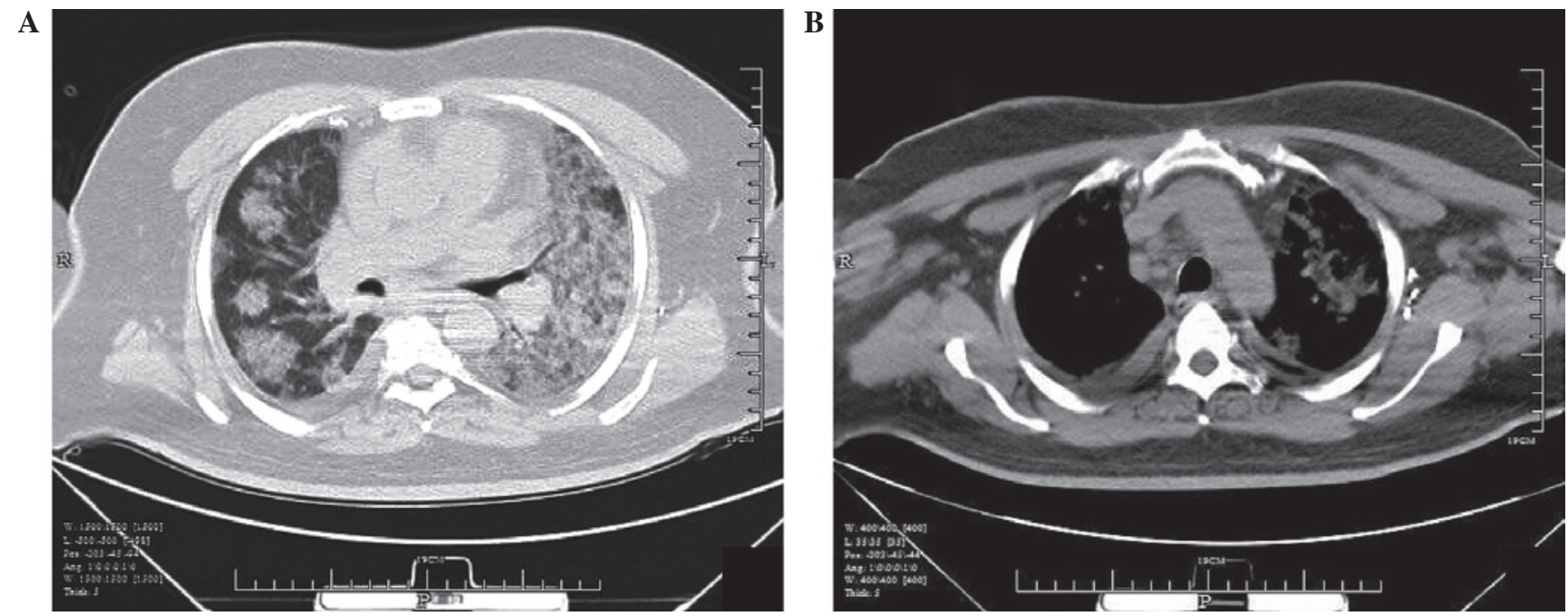

Figure 1. Computerized tomography scans of the chest at a lung-window setting indicated (A) unclear lung fields associated with diffuse pathological changes in both lungs and signs of severe pulmonary infection. The mediastinal-window setting showed (B) pleural effusion multiple lymphadenectasis in retrocaval and para-aortic arch area.

no expectoration, chest pain, hemoptysis or oliguresis were reported, and the patient did not receive regular therapy. Within the 6 days after the onset of symptoms, cough and chest tightness became progressively worse, and the edema of both lower limbs appeared. The patient's medical history indicated that the patient suffered from diabetes within the past 20 years, which was treated with insulin, coronary heart disease performed 10 years before presentation to our hospital and chronic diabetic kidney insufficiency over the last 4 years. In addition, the patient reported that chest tightness and tachypnea had occurred soon after walking for 100 steps within the past 10 years. Family history was unremarkable.

On admission, the patient was found to have fever (oral temperature of $38.5^{\circ} \mathrm{C}$; normal $36.3-37.3^{\circ} \mathrm{C}$ ) and slight tachypnea (25 respirations/min; normal 12-20 respirations/min), with a heart rate (HR) of 60 beats/min (normal $60-100$ beats/min), a pulse of 60/min (normal 60-100/min) and blood pressure (BP) of 180/90 mmHg (normal 90/60 mmHg-120/80 mmHg). The patient had an oxygen saturation of 90\% (normal 98-100\%) on air and presented cyanosis in the lips. Cardiovascular examinations Laboratory examinations revealed an irregular heart rhythm, however, no evident pathological murmur was observed at the auscultatory valve areas and no distension of the jugular vein was observed at a semireclining position. Crude breath sounds of bilateral lung were heard on chest auscultation, without marked dry and moist rales. Other physical examinations included the abdominal bulge in the absence of tenderness and rebound, unsatisfactory palpation of liver and spleen, borborygmus at a rate of $4 / \mathrm{min}$ with negative shifting dullness, as well as mild edema of the lower limbs. Neurogenic examination showed no abnormalities. The admitting diagnosis was severe pneumonia due to suspected MERS, respiratory and heart failure, acid base imbalance and electrolyte disturbances, diabetes, chronic kidney insufficiency and hypertension.

Laboratory examinations by a Sysmex XE-2100 hematology analyzer (Sysmex Corporation, Kobe, Japan) revealed the following levels: Hemoglobin, $96 \mathrm{~g} / 1$ (depressed; normal,
130-175 g/l); white blood cells, $10.2 \times 10^{9} / 1$ [(77.6\% neutrophils, $13.7 \%$ lymphocytes and $8.7 \%$ monocytes); elevated; normal, 3.5-9.5 x 10\% $/ 1$ (40.0-75.0\% neutrophils)]; and C-reactive protein, $>200 \mathrm{mg} / \mathrm{dl}$ (elevated; normal, 0-80 mg/dl). In addition, blood gas examination by an SC-501 automated blood gas analyzer (Radiometer, Copenhagen, Denmark) demonstrated the following results: $\mathrm{pH} 7.42$ (normal 7.35-7.45); partial pressure of oxygen, $65.8 \mathrm{mmHg}$ (depressed; normal 80-100 mmHg); partial pressure of carbon dioxide, $30.8 \mathrm{mmHg}$ (depressed; normal 35-45 $\mathrm{mmHg}$ ); BE, $4 \mathrm{mmol} / 1$ (elevated; normal -3-+3 mmol/1); bicarbonate level, $19.8 \mathrm{mmol} / \mathrm{l}$ (depressed normal 22-27 mmol/1); and lactic acid level, $2.10 \mathrm{mmol} / 1$ (elevated; normal 0.44-1.78 mmol/l). Furthermore, biochemical analyses by a fully automatic biochemical analyzer (Architect c16000; Abbott Diagnostics, Lake Forest, IL, USA) showed the following results: Aspartate aminotransferase, $85 \mathrm{U} / 1$ (elevated; normal 9-50 U/1); lactate dehydrogenase, $369 \mathrm{U} / 1$ (elevated; normal 0-200 U/1); blood potassium, $5.01 \mathrm{mmol} / 1$ (normal, 3.50-5.50 mmol/1); blood sodium, $128 \mathrm{mmol} / 1$ (depressed; normal 137-147 mmol/1); blood glucose, $16.89 \mathrm{mmol} / \mathrm{l}$ (elevated; normal 3.60-6.10 mmol/l); creatinine, $224 \mu \mathrm{mol} / \mathrm{l}$ (elevated; normal 15-110 $\mu \mathrm{mol} / 1$ ); blood urea nitrogen, $20.62 \mathrm{mmol} / \mathrm{l}$ (elevated normal 1.10-8.30 mmol/1). Besides, troponin was assessed as weakly positive (normal negative) by a troponin analyzer (Zhe Jiang Ikon Biotechnology Co., Ltd, Zhejiang, China), and the brain natriuretic peptide was detected 4,567 pg/ml (elevated; normal 0.00-125 pg/ml) by a Cobas 6000 biochemistry analyzer (Roche, Berlin, Germany).

Computerized tomography (CT) of the chest at lung-window setting demonstrated unclear lung fields associated with diffuse pathological changes in the two lungs and signs of severe pulmonary infection (Fig. 1A). In addition, pleural effusion and multiple lymphadenectasis in retrocaval and para-aortic arch area were observed at mediastinal-window setting (Fig. 1B). Blood samples were obtained for the detection of procalcitonin (PCT), and syphilis or human immunodeficiency virus (HIV) infection. Blood, sputum and pharynx swab samples were also collected and sent to the Provincial Center for Disease 
Control (Hangzhou, Zhejiang, China) in order to detect viruses or bacteria. Antibodies to syphilis and HIV in the blood were found to be negative at day 2 after admission. In addition, the serum PCT concentration of the patient was $4.73 \mu \mathrm{g} / \mathrm{l}$ at day 2 after admission and $7.23 \mu \mathrm{g} / \mathrm{l}$ (normal $<0.06 \mu \mathrm{g} / \mathrm{l}$ ) at day 3 after admission. All repeat blood culture sets did not yield any growth. Diagnosis of numerous viruses by nucleic acid detection was also negative at 2 days after admission, including absence of infection with MERS-coronavirus (MERS-CoV), flu viruses of type A and B (including the H1N1 and H7N9 subtypes of influenza A), Mycoplasma pneumoniae, Chlamydia pneumoniae, respiratory adenovirus, respiratory syncytial viruses and various parainfluenza viruses (types I, II and III). In addition, galactomannan (GM) detection in the blood was negative. A sputum smear showed a preliminary result of fungal infection, and the culture was repeated three times, indicating a positive result for $C$. krusei infection 4 days after admission. Therefore, the diagnosis of $C$. krusei pneumonia was established, but specific treatment for $C$. kruse $i$ was not administered due to the patient succumbing to the disease 2 days after admission.

Initially, the patient was isolated and received $95-98 \%$ oxygen at atmospheric pressure at a rate of $61 / \mathrm{min}$ through a nasal catheter for $24 \mathrm{~h}$ after admission to the hospital. Due to the history of diabetes, a poor therapeutic effect for community infection, an auxiliary and radiological examination, the patient was administered sulbactam sodium/cefoperazone sodium (1:1) by injection $(3.0 \mathrm{~g} /$ time, which is the total dose used for injection; Pfizer Pharmaceuticals Ltd., Liaoning, China) every $12 \mathrm{~h}$ for antibacterial infection treatment before the definite diagnosis. Oral administration of oseltamivir capsules ( $75 \mathrm{mg} / \mathrm{time}$; Roche, Bale, Switzerland) was performed twice a day for antiviral infection treatment. Intravenous administration of medaron (Pfizer Manufacturing Belgium NV, Puurs, Belgium) at a dose of $80 \mathrm{mg} /$ time was also started empirically for anti-inflammatory treatment, as well as $750 \mathrm{ml}$ fluid infusion. However, no significant improvement was noted, and the condition of the patient deteriorated, with cardiac and respiratory arrest occurring one day after admission. Cardiopulmonary resuscitation was immediately performed, and the patient recovered weak respiration with an assisted respirator. The $\mathrm{BP}$ of the patient at this time was $70 / 50 \mathrm{mmHg}$, which was raised and stabilized at $110 / 80 \mathrm{mmHg}$ after adjusting the speed of fluid infusion, monitoring central venous pressure, and minipump infusion (4-20 ml/h, adjusted according to the BP) of vasoactive agents, including noradrenaline $(10 \mathrm{mg})$ and normal saline $(50 \mathrm{ml})$. Furthermore, a low HR of $30-40$ beats/min was recorded and thus cardiotonic therapy was administered, which consisted of adrenalin injection $(10 \mathrm{mg})$ and normal saline $(50 \mathrm{ml})$ at a minipump maintenance dose of 4-20 ml/h (adjusted according to the BP and HR). Furthermore, the renal function of the patient was aggravated, with the appearance of oliguria and anuresis. In spite of continuing renal replacement therapy, the patient succumbed to the infection 2 days after admission.

\section{Discussion}

C. krusei is an opportunistic pathogen of normal human microbial flora localized in the skin, mucous membranes and digestive tract, and it may cause life-threatening invasive infections (17). Despite not being the most frequently isolated species of Candida in infected patients, C. kruse i is an invasive infection with growing incidence (18). Candida pneumonia, a rare infection associated with high mortality, should always be considered in patients presenting cough, expectoration of purulent secretions, occasional hemoptysis and invariably hypoxemia (8).

In the present study, the patient developed paroxysmal cough, accompanied by chest tightness, tachypnea and slight fever. However, pulmonary infection due to C. krusei in the current patient was difficult to diagnose, and initially MERS was suspected. MERS is caused by the novel MERS-CoV pathogen, a viral respiratory infection first reported in the Saudi Arabian peninsula in 2012 (16). This infection is characterized by acute respiratory infection, and develops into respiratory failure, acute respiratory distress syndrome and multiple organ failure, particularly renal failure (19). The common symptoms of MERS also include fever, cough and shortness of breath. In addition, the majority of MERS patients present underlying comorbid medical disorders, including diabetes, hypertension, chronic cardiac disease and chronic renal disease (20). The patient of the current study presented similar symptoms to the aforementioned MERS symptoms; however, MERS was excluded by negative detection of the virus through the nucleic acid detection method.

Histopathological examination of tissue specimens obtained by invasive procedures is considered as the gold standard for diagnosis of Candida pneumonia (21). Considering the difficulty in performing biopsy, repeat blood and sputum cultures are typically conducted (4), and the patient and his family disallowed for invasive operation because of their belief. Isolation of $C$. krusei from the sputum is almost always considered to represent colonization of the respiratory tract. However, this diagnostic method presents great hysteresis. Currently, PCT and GM tests are used as auxiliary examination methods for the diagnosis of fungal infection $(22,23)$. Serum PCT levels are $0.1 \mu \mathrm{g} / 1$ in healthy individuals, and increased secretion is observed under bacterial infection $(24,25)$. In the present case, the repeatedly high PCT concentrations supported a diagnosis of $C$. krusei infection, although a false-negative result was obtained in the GM test, which was not considered to be inaccurate since it was not conducted successionally and repeatedly.

Candida invasive infection usually affects immunocompromised patients or those receiving broad-spectrum antibiotic therapy. As reported in previous studies, solid organ transplant $(3,26)$, hematological malignancies $(27)$, esophageal perforation (28) and diabetes (29) appear to facilitate C. krusei pneumonia and empyema (11). Similarly, the patient of the current study presented a long-term medical history of diabetes, coronary heart disease and chronic diabetic kidney insufficiency, but did not receive administration of hormone therapy and antibiotics. The pathogenetic condition progressed severely and fast, which is inconsistent with common fungal pneumonia, and thus resulted in misdiagnosis.

In conclusion, the present case provides several learning points on Candida pneumonia. The diagnosis of Candida pneumonia should be strongly considered in the presence of growth of Candida pathogens from a sputum culture and a suggestive CT image. In addition, tumescent diaphragmatic 
lymph nodes may be an important symptom of Candida pneumonia. Finally, treatment should be initiated immediately in order to improve tissue oxygenation, restore cardiovascular function and improve other organ functions. This study, to a certain extent, would provide guidance or some experiences to the physician in diagnosing Candida pneumonia in the clinic.

\section{Acknowledgements}

The authors would like to thank the Zhejiang Provincial Center for Disease Control and Prevention Clinical Laboratory, and the Zhejiang First Hospital Infection Department Laboratory that conducted comprehensive etiology detection for the current patient.

\section{References}

1. Haron E, Vartivarian S, Anaissie E, Dekmezian R and Bodey GP: Primary Candida pneumonia: Experience at a large cancer center and review of the literature. Medicine (Baltimore) 72: 137-142, 1993.

2. Von Eiff M, Roos N, Fegeler W, Von Eiff C, Schulten R, Hesse M, Zühlsdorf $\mathrm{M}$ and Van de Loo J: Hospital - acquired candida and aspergillus pneumonia - diagnostic approaches and clinical findings. J Hosp Infect 32: 17-28, 1996.

3. Petrocheilou-Paschou V, Georgilis K, Kontoyannis D, Nanas J, Prifti H, Costopoulos H and Stamatelopoulos S: Pneumonia due to Candida krusei. Clin Microbiol Infect 8: 806-809, 2002.

4. Carvajal C, Rello J and Lipman J: Candida pneumonia in patients with hematological neoplasia. In: Pulmonary Involvement in Patients with Hematological Malignancies. Azoulay E (ed.). Springer, Berlin, pp349-356, 2011.

5. Masur H, Rosen PP and Armstrong D: Pulmonary disease caused by Candida species. Am J Med 63: 914-925, 1977.

6. von Rosenstein N R: The diseases of children and their remedies. The Classics of Medicine Library, Wenneberg and Nordstrom, Stockholm 1984

7. Castellani A: Notes on certain bronchomycoses which may simulate pulmonary tuberculosis. Am Rev Tuberc 16: 1927-1928, 1927.

8. Mohsenifar Z, Chopra SK, Johnson BL and Simmons DH: Candida pneumonia: Experience with 20 patients. West J Med 131: 196-200, 1979.

9. Schröter GP, Hoelscher M, Putnam CW, Porter KA and Starzl TE: Fungus infections after liver transplantation. Ann Surg 186: 115-122, 1977.

10. Buff SJ, McLelland R, Gallis HA, Matthay R and Putman CE Candida albicans pneumonia: radiographic appearance. Am J Roentgenol 138: 645-648, 1982.

11. Imtiaz T, Thomson F, Innes A, du Toit FC and Bal AM: Candida krusei bronchopneumonia with nodular infiltrates in a patient with chronic renal failure on haemodialysis - case report and review of literature. Mycoses 54: e611-e614, 2011.

12. Meersseman W, Lagrou K, Spriet I, Maertens J, Verbeken E, Peetermans W and Van Wijngaerden E: Significance of the isolation of Candida species from airway samples in critically ill patients: a prospective, autopsy study. Intens Care Med 35: 1526-1531, 2009.

13. Gogia P: Pulmonary fungal infections. Current Medicine Research and Practice 5: 221-227, 2015.

14. Kontoyiannis D, Reddy B, Torres H, Luna M, Lewis R, Tarrand J, Bodey G and Raad I: Pulmonary candidiasis in patients with cancer: an autopsy study. Clin Infect Dis 34: 400-403, 2002.
15. Rodriguez L, Anaissie E and Rex J: Pneumonia due to Candida species. In: Sarosi GA, Davies SF, eds. Fungal disease of the lung, 3rd edn. Lippincott Williams \& Wilkins, Philadelphia, pp115-122, 2000.

16. Zaki AM, Van Boheemen S, Bestebroer TM, Osterhaus AD and Fouchier RA: Isolation of a novel coronavirus from a man with pneumonia in Saudi Arabia. N Engl J Med 367: 1814-1820, 2012.

17. Galván B and Mariscal F: Epidemiology of candidemia in ICU. Rev Iberoam Micol 23: 12-15, 2006 (In Spanish).

18. Fidan I, Yesilyurt E, Kalkanci A, Aslan SO, Sahin N, Ogan MC and Dizbay M: Immunomodulatory effects of voriconazole and caspofungin on human peripheral blood mononuclear cells stimulated by Candida albicans and Candida krusei. Am J Med Sci 348: 219-223, 2014.

19. WHO MERS-CoV Research Group: State of knowledge and data gaps of Middle East respiratory syndrome coronavirus (MERS-CoV) in humans. PLoS Curr 5: 2013.

20. Assiri A, Al-Tawfiq JA, Al-Rabeeah AA, Al-Rabiah FA, Al-Hajjar S, Al-Barrak A, Flemban H, Al-Nassir WN, Balkhy HH, Al-Hakeem RF, et al: Epidemiological, demographic, and clinical characteristics of 47 cases of Middle East respiratory syndrome coronavirus disease from Saudi Arabia: A descriptive study. Lancet Infect Dis 13: 752-761, 2013.

21. Vélez L, Correa LT, Maya MA, Mejía P, Ortega J, Bedoya V and Ortega H: Diagnostic accuracy of bronchoalveolar lavage samples in immunosuppressed patients with suspected pneumonia: Analysis of a protocol. Respir Med 101: 2160-2167, 2007.

22. Musher B, Fredricks D, Leisenring W, Balajee SA, Smith C and Marr KA: Aspergillus galactomannan enzyme immunoassay and quantitative PCR for diagnosis of invasive aspergillosis with bronchoalveolar lavage fluid. Journal of clinical microbiology 42: 5517-5522, 2004.

23. Charles PE, Castro C, Ruiz-Santana S, León C, Saavedra P and Martín E: Serum procalcitonin levels in critically ill patients colonized with Candida spp: new clues for the early recognition of invasive candidiasis? Intensive care medicine 35: 2146-2150, 2009.

24. Groselj-Grenc M, Ihan A, Pavcnik-Arnol M, Kopitar AN, Gmeiner-Stopar T and Derganc M: Neutrophil and monocyte CD64 indexes, lipopolysaccharide-binding protein, procalcitonin and C-reactive protein in sepsis of critically ill neonates and children. Intensive Care Med 35: 1950-1958, 2009.

25. Christ-Crain M, Jaccard-Stolz D, Bingisser R, Gencay MM, Huber PR, Tamm M and Müller B: Effect of procalcitonin-guided treatment on antibiotic use and outcome in lower respiratory tract infections: Cluster-randomised, single-blinded intervention trial. Lancet 363: 600-607, 2004.

26. Bonatti H, Stelzmueller I, Berger N, Lechner M, Grif K, Geltner C, Margreiter R and Lass-Flörl C: Infections caused by Candida krusei in five transplant and two surgical patients. Surg Infect 10: 265-271, 2009.

27. Speletas M, Vyzantiadis T-A, Kalala F, Plastiras D, Kokoviadou K, Antoniadis A and Korantzis I: Pneumonia caused by Candida krusei and Candida glabrata in a patient with chronic myeloid leukemia receiving imatinib mesylate treatment. Med Mycol 46: 259-263, 2008.

28. Cascio A, Barone M, Micali V, Iaria C, Delfino D, David A, Monaco M and Monaco F: On a fatal case of Candida krusei pleural empyema in a pregnant woman with spontaneous esophagus perforation. Mycopathologia 169: 451-455, 2010.

29. Kofteridis DP, Mantadakis E, Karatzanis AD, Bourolias CA, Papazoglou G, Velegrakis GA and Samonis G: Non-Candida albicans Candida mediastinitis of odontogenic origin in a diabetic patient. Med Mycol 46: 345-348, 2008. 